\title{
Unification of gravitation and electromagnetic force(First Portion)
}

This paper was downloaded from TechRxiv (https://www.techrxiv.org).

\section{LICENSE}

CC BY 4.0

SUBMISSION DATE / POSTED DATE

$15-10-2021 / 05-11-2021$

CITATION

shengming, Zheng (2021): Unification of gravitation and electromagnetic force(First Portion). TechRxiv. Preprint. https://doi.org/10.36227/techrxiv.16817275.v2

$\mathrm{DOI}$

10.36227/techrxiv.16817275.v2 


\section{Unification of gravitation and electromagnetic force (First Portion)}

Zheng shengming (郑胜明)（Physicsworks@tom.com）

The contents of this article fall within Fields, Wave and Electromagnetics; Photonics and Electrooptics area of scope of TechRxiv, part of it are published in my book (ISBN: 9789881767592) but is not published in journal. For convenience readers, I revise and submit it to here

\begin{abstract}
Since electron has been discovered, people have been thinking it possess one electric charge, the nuclear possess contrary charge. Based on the provided new discovery that moving photons do create force, $I$ have calculated the number $Z$ of elements, discovered that the number $Z$ of elements can be calculated by frequency of $x$-ray, and the atomic weight can also be calculated by frequency of $x$-ray. This method of calculated way shows the essence of electric charge, and shows the essence of gravitational mass, from this way; here in first time provide one fact show the unification of gravitation and electromagnetic force.
\end{abstract}

Keywords: frequency of $\mathrm{x}$-ray, force, gravitation, electromagnetic force, unification.

\section{INTRODUCTION}

Since Newton discovered universal gravitation [1], people have been thinking that the origin of gravitation only relate to the gravitational mass, and define gravitational mass in the expression of the gravitation without investigating other origin theories. While during the process of investigating the origin of gravitation, my experiments showed that moving photons produce gravitation. This discovery reveals the origin of gravitation. Meanwhile I found that the atomic weight can be calculated by frequency of $x$-ray[2]. On the other hand, I have also found that the number $\mathrm{Z}$ of elements can also be calculated by frequency of $\mathrm{x}$-ray. By these experiments and calculations, to show that the electromagnetic force and gravitation are generated from the same origin. Their essence is the same. So we can comprehend the meaning of the gravitational mass which defined by Newton and Einstein and the meaning of charge which defined by Coulomb and Franklin. This shows the unification of gravitation and electromagnetic force.

\section{Chapter.1. Moving photon generate force}

The experimental devices are indication in picture $\mathbf{a}, \mathbf{b}, \mathbf{c}$ of Figure1. The process of experiment is below: first the light beam $\mathbf{L}$ is separated into 2 parts by a ring as position in picture c, $\mathbf{d}$ of Figure1. Light beam $\mathbf{L}$ then become two new light beams $\mathbf{P}$ and $\mathbf{0}$, as shown in picture $\mathbf{d}$ of Figure1. Along with light moving forward, the five light beams possessing highest intensity in the light beam $\mathbf{P}$ with biggest attractive force have been obviously attracting light beam $\mathbf{O}$ to become a pentagon(see picture 1-12 of Figure1). Only the five spots of light beams $\mathbf{O}$ that corresponding five points of light beams which possessing highest intensity in light beam $\mathbf{P}$, have been moving to contact, and become gradually link to together. Note: at first light beam $\mathbf{P}$ and light beam $\mathbf{O}$ do not contact at all. It is impossible that this contact action can occurs in the light wave theory. This action is not an effect of wave interaction. On the contrary, it is indicating an effect of moving photons create gravitation. (See picture 1-12 of Figure1)

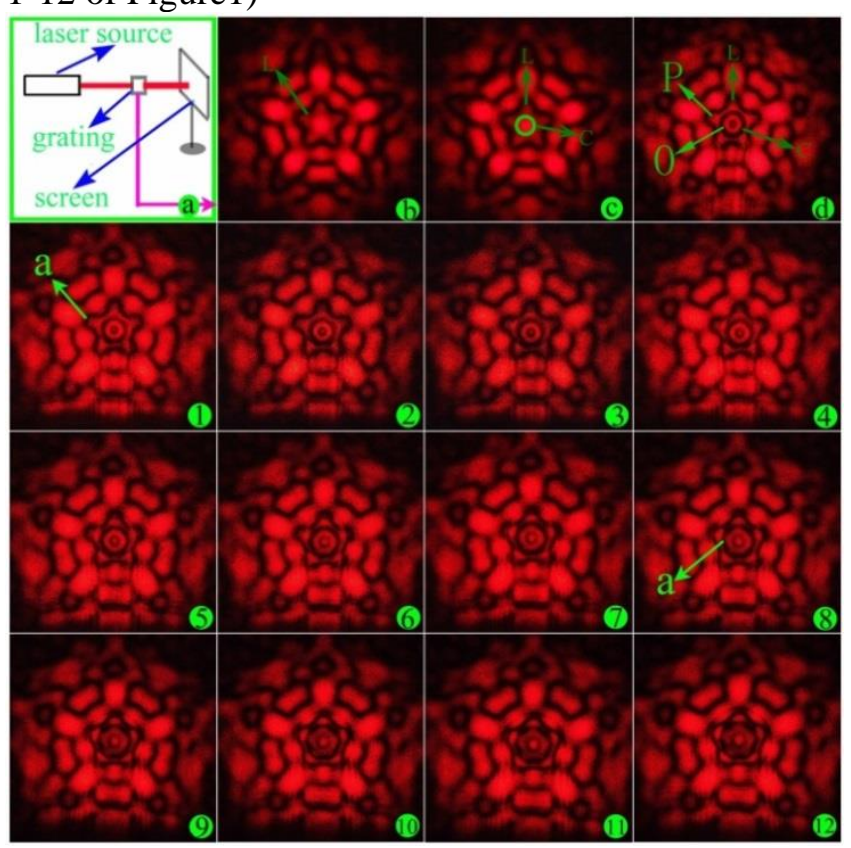

Figure1. Picture $\mathbf{b}$ shows the device of the experiment. Picture $\mathbf{c}, \mathbf{d}$ shows the site of ring in light beam 0 , and pictures 1-12showing that the form of light beam $O$ has been changed from a circular shape to a pentagon by moving photon creates gravitation.

In order to verify this phenomenon is brought on by gravitation, the second experiment is performed. The experiment device is shown in picture a of Figure2. Picture b, $\mathbf{c}$ in Figure2 show 
that other light beams do not move forward and that only light beam $\mathrm{O}$ is allowed to move forward; under this status, light beam 0 has been keeping its circular shape. This phenomenon is shown in pictures 1-12 of Figure2. This result demonstrates that if there was no other light beam, the light beam 0 would not accept the foregone gravitation.

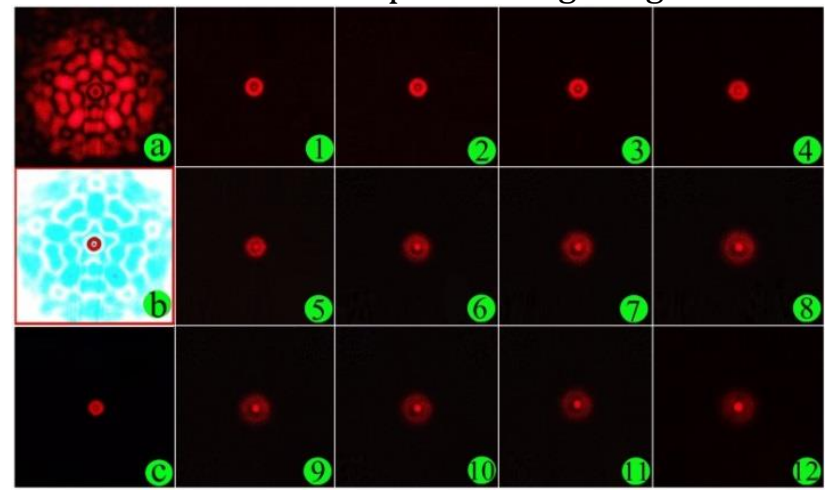

Figure2. This experiment shows that light beam 0 does not change its circular shape when there are no other light beams moving forward.

In comparison with experiment 1 , it is appearing that the light beam 0 keeps its circular shape in unchanged form when there are no other light beams moving forward. From this experiment, the light beam 0 does not appear before phenomenon of gravitation acted upon it become, it is confirm that this is only appeared with interaction force indicating gravitation brought about it, thus we find that moving photons create gravitation.

\section{Chapter.2. The quantitative experiment}

In before article: Gravitation origin ${ }^{[2]}$, we get below formula: $\vec{F}_{a}=\frac{m_{1} m_{2} \times \vec{v}_{1} \times\left(\vec{v}_{2} \times \vec{r}_{12}\right)}{4 \pi \Theta \vec{r}_{12}^{3}} G_{a}$

About describing of this effect, see Gravitation origin [2], here do not repeat it.

\section{Chapter.3. The essence of electric charge}

According to above discoveries and formula:

$\vec{F}_{a}=\frac{m_{1} m_{2} \times \vec{v}_{1} \times\left(\vec{v}_{2} \times \vec{r}_{12}\right)}{4 \pi \Theta \vec{r}_{12}^{3}} G_{a}$

we can get a formula that can calculate the nuclear charge of element, see formula: $Q_{Z}=\sqrt{\frac{c}{\bar{\lambda}}+Z b} \times$ $\left(1-\left(A_{m}-Z\right) k_{1}\right) k_{2}$ Where, $Q_{Z}$ is the nuclear charge of element, $A_{m}$ is the atomic weight of element, $Z$ is the atomic number, $\lambda_{k 1}, \lambda_{k 2}$ are the first and second wavelength of X-Ray Emission in $k, \bar{\lambda}$ is the mean wavelength of these two wavelengths of X-Ray Emission. $b, k_{1}, k_{2}$ are all constant. The result of test about this formula see below table 1, Table2 [3],[4] .

$\mathrm{K}_{c} 1=\mathrm{KL} 3=1 s 1 / 2-2 p 3 / 2$,

$\mathrm{K}_{c} 2=\mathrm{KL} 2=1 s 1 / 2-2 p 1 / 2^{[3],[4]}$.

$\bar{\lambda}=\frac{\delta \lambda_{1}+\lambda_{2}}{2} \times 10^{-10}$

For example about Li: $Q_{Z}=\sqrt{\frac{c}{\lambda}+Z b} \times\left(1-\left(A_{m}-Z\right) k_{1}\right) \times k_{2}$ $=\sqrt{\frac{3311^{8}}{23419 \times 10^{10}}+3 \times 2.72641 \times 10^{15}} \times\left(1-(6.941-3) \times 5 \times 10^{-4}\right) \times 2.075 \times 10^{-8}$ $=3$. Other element number or nuclear charge of element can also be calculated by this formula. See above table1. This formula testifies that the nuclear charge of element can be calculated by wavelength of X-Ray of element, thus this formula indicate the essence of nuclear charge of element, namely reveal the essence of electric charge.

Table $1^{[3],[4],[5],[6] \text { : }}$

\begin{tabular}{|c|c|c|c|c|c|c|c|c|c|c|c|}
\hline Z & sym & omic w & $A_{m}-Z$ & $\lambda_{41} \times 10^{-10}$ & $\lambda_{12} \times 10^{-10}$ & $\delta$ & $\bar{\lambda}=\frac{a_{0}+\lambda_{2}}{2} \times 10^{-10}$ & $b$ & $k_{l}$ & $k_{2}$ & 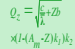 \\
\hline 3 & $\mathrm{Li}$ & 6.941 & 3.941 & 241.686 & 226.456 & 1.001 & 234.192 & $2.72641 \times 10^{15}$ & $5 \times 10^{-4}$ & $2.075 \times 10^{-8}$ & 3.000 \\
\hline 4 & $\mathrm{Be}$ & 9.012 & 5.012 & 114.272 & 111.698 & 1.007 & 113.379 & $2.72641 \times 10^{15}$ & $5 \times 10^{-4}$ & $2.075 \times 10^{-8}$ & 4.000 \\
\hline 5 & B & 10.811 & 5.881 & 67.6400 & 65.9495 & 1.005 & 66.9638 & $2.72641 \times 10^{15}$ & $5 \times 10^{-4}$ & $2.075 \times 10^{-8}$ & 5.000 \\
\hline 6 & C & 12.011 & 6.011 & 44.7600 & 43.6813 & 1.002 & 44.2654 & $2.72641 \times 10^{15}$ & $5 \times 10^{-4}$ & $2.075 \times 10^{-8}$ & 6.000 \\
\hline 7 & $\mathrm{~N}$ & 14.006 & 7.006 & 31.5966 & 30.9899 & 1.006 & 31.3880 & $2.72641 \times 10^{15}$ & $5 \times 10^{-4}$ & $2.075 \times 10^{-8}$ & 7.000 \\
\hline 8 & 0 & 15.999 & 7.999 & 23.6207 & 23.3186 & 0.996 & 23.4224 & $2.72641 \times 10^{15}$ & $5 \times 10^{-4}$ & $2.075 \times 10^{-8}$ & 8.000 \\
\hline 9 & $\mathrm{~F}$ & 18.998 & 9.998 & 18.2000 & 18.0499 & 1.000 & 18.1250 & $2.72641 \times 10^{15}$ & $5 \times 10^{-4}$ & $2.075 \times 10^{-8}$ & 9.000 \\
\hline 10 & $\mathrm{Ne}$ & 20.179 & 10.179 & 14.6105 & 14.3023 & 1.001 & 14.4637 & $2.72641 \times 10^{15}$ & $5 \times 10^{-4}$ & $2.075 \times 10^{-8}$ & 10.00 \\
\hline 11 & $\mathrm{Na}$ & 22.989 & 11.989 & 11.9102 & 11.6174 & 1.004 & 11.7876 & $2.72641 \times 10^{15}$ & $5 \times 10^{-4}$ & $2.075 \times 10^{-8}$ & 11.00 \\
\hline 12 & $\mathrm{Mg}$ & 24.305 & 12.305 & 9.80700 & 9.80700 & 1.000 & 9.80700 & $2.72641 \times 10^{15}$ & $5 \times 10^{-4}$ & $2.075 \times 10^{-8}$ & 12.00 \\
\hline 13 & $\mathrm{Al}$ & 26.981 & 13.981 & 8.27067 & 8.27067 & 1.000 & 8.27067 & $2.72641 \times 10^{15}$ & $5 \times 10^{-4}$ & $2.075 \times 10^{-8}$ & 13.00 \\
\hline 14 & $\mathrm{Si}$ & 28.085 & 14.085 & 7.07677 & 7.07677 & 1.001 & 7.08030 & $2.72641 \times 10^{15}$ & $5 \times 10^{-4}$ & $2.075 \times 10^{-8}$ & 14.00 \\
\hline 15 & P & 30.973 & 15.973 & 6.11663 & 6.11663 & 1.001 & 6.11968 & $2.72641 \times 10^{15}$ & $5 \times 10^{-4}$ & $2.075 \times 10^{-8}$ & 15.00 \\
\hline 16 & S & 32.065 & 16.065 & 5.35329 & 5.34077 & 1.002 & 5.35038 & $2.72641 \times 10^{15}$ & $5 \times 10^{4}$ & $2.075 \times 10^{-8}$ & 16.00 \\
\hline 17 & $\mathrm{Cl}$ & 35.453 & 18.453 & 4.71100 & 4.69567 & 1.001 & 4.70569 & $2.72641 \times 10^{15}$ & $5 \times 10^{-4}$ & $2.075 \times 10^{-8}$ & 17.00 \\
\hline 18 & $\mathrm{Ar}$ & 39.948 & 21.948 & 4.19180 & 4.19180 & 0.993 & 4.16245 & $2.72641 \times 10^{15}$ & $5 \times 10^{-4}$ & $2.075 \times 10^{-8}$ & 18.00 \\
\hline 19 & K & 39.098 & 20.098 & 3.74140 & 3.72060 & 1.000 & 3.73100 & $2.72641 \times 10^{15}$ & $5 \times 10^{-4}$ & $2.075 \times 10^{-8}$ & 19.00 \\
\hline 20 & $\mathrm{Ca}$ & 40.078 & 20.078 & 3.36166 & 3.34013 & 1.003 & 3.35593 & $2.72641 \times 10^{15}$ & $5 \times 10^{-4}$ & $2.075 \times 10^{-8}$ & 20.00 \\
\hline 21 & Sc & 44.956 & 23.955 & 3.03090 & 3.03090 & 0.997 & 3.02181 & $2.72641 \times 10^{15}$ & $5 \times 10^{-4}$ & $2.075 \times 10^{-8}$ & 21.00 \\
\hline 22 & Ti & 47.867 & 25.876 & 2.74851 & 2.74851 & 0.997 & 2.74026 & $2.72641 \times 10^{15}$ & $5 \times 10^{-4}$ & $2.075 \times 10^{-8}$ & 22.00 \\
\hline 23 & V & 50.941 & 27.941 & 2.50356 & 2.50356 & 0.997 & 2.49605 & $2.72641 \times 10^{15}$ & $5 \times 10^{-4}$ & $2.075 \times 10^{-8}$ & 23.00 \\
\hline 24 & $\mathrm{Cr}$ & 51.996 & 27.996 & 2.28970 & 2.28970 & 0.999 & 2.28741 & $2.72641 \times 10^{15}$ & $5 \times 10^{-4}$ & $2.075 \times 10^{-8}$ & 24.00 \\
\hline 25 & $\mathrm{Mn}$ & 54.938 & 29.938 & 2.10180 & 2.10180 & 0.999 & 2.09969 & $2.72641 \times 10^{15}$ & $5 \times 10^{-4}$ & $2.075 \times 10^{-8}$ & 25.00 \\
\hline 26 & $\mathrm{Fe}$ & 55.845 & 29.845 & 1.93998 & 1.93735 & 1.000 & 1.93866 & $2.72641 \times 10^{15}$ & $5 \times 10^{-4}$ & $2.075 \times 10^{-8}$ & 26.00 \\
\hline 27 & Co & 58.933 & 31.933 & 1.79285 & 1.78896 & 1.000 & 1.79091 & $2.72641 \times 10^{15}$ & $5 \times 10^{4}$ & $2.075 \times 10^{-8}$ & 27.00 \\
\hline 28 & $\mathrm{Ni}$ & 58.693 & 30.693 & 1.66179 & 1.66179 & 1.001 & 1.66345 & $2.72641 \times 10^{15}$ & $5 \times 10^{-4}$ & $2.075 \times 10^{-8}$ & 28.00 \\
\hline 29 & $\mathrm{Cu}$ & 63.546 & 34.546 & 1.54440 & 1.54056 & 1.001 & 1.54325 & $2.72641 \times 10^{15}$ & $5 \times 10^{4}$ & $2.075 \times 10^{-8}$ & 29.00 \\
\hline 30 & $\mathrm{Zn}$ & 65.409 & 35.409 & 1.43900 & 1.43515 & 1.002 & 1.43851 & $2.72641 \times 10^{15}$ & $5 \times 10^{-4}$ & $2.075 \times 10^{-8}$ & 30.02 \\
\hline 31 & Ga & 69.723 & 38.723 & 1.34138 & 1.34138 & 1.000 & 1.34138 & $2.72641 \times 10^{15}$ & $5 \times 10^{-4}$ & $2.075 \times 10^{-8}$ & 31.00 \\
\hline 32 & Ge & 72.641 & 40.640 & 1.25405 & 1.25405 & 1.000 & 1.25405 & $2.72641 \times 10^{15}$ & $5 \times 10^{-4}$ & $2.075 \times 10^{-8}$ & 32.00 \\
\hline 33 & As & 74.921 & 41.921 & 1.17588 & 1.17588 & 1.001 & 1.17705 & $2.72641 \times 10^{15}$ & $5 \times 10^{-4}$ & $2.075 \times 10^{-8}$ & 33.00 \\
\hline 34 & Se & 78.963 & 44.960 & 1.10477 & 1.10477 & 0.999 & 1.10366 & $2.72641 \times 10^{15}$ & $5 \times 10^{-4}$ & $2.075 \times 10^{-8}$ & 34.00 \\
\hline 35 & $\mathrm{Br}$ & 79.904 & 44.904 & 1.03974 & 1.03974 & 1.001 & 1.04077 & $2.72641 \times 10^{15}$ & $5 \times 10^{-4}$ & $2.075 \times 10^{-8}$ & 35.00 \\
\hline 36 & $\mathrm{Kr}$ & 83.798 & 47.798 & 0.98010 & 0.98010 & 0.999 & 0.98000 & $2.72641 \times 10^{15}$ & $5 \times 10^{-4}$ & $2.075 \times 10^{-8}$ & 36.00 \\
\hline 37 & $\mathrm{Rb}$ & 85.467 & 48.467 & 0.92550 & 0.92550 & 1.000 & 0.92550 & $2.72641 \times 10^{15}$ & $5 \times 10^{-4}$ & $2.075 \times 10^{-8}$ & 37.00 \\
\hline 38 & $\mathrm{Sr}$ & 87.621 & 49.620 & 0.87529 & 0.87529 & 1.001 & 0.87616 & $2.72641 \times 10^{15}$ & $5 \times 10^{4}$ & $2.075 \times 10^{-8}$ & 38.00 \\
\hline 39 & Y & 88.905 & 49.905 & 0.83071 & 0.83071 & 1.000 & 0.83070 & $2.72641 \times 10^{15}$ & $5 \times 10^{-4}$ & $2.075 \times 10^{-8}$ & 39.00 \\
\hline 40 & $\mathrm{Zr}$ & 91.224 & 51.224 & 0.79012 & 0.78595 & 1.000 & 0.78803 & $2.72641 \times 10^{15}$ & $5 \times 10^{-4}$ & $2.075 \times 10^{-8}$ & 40.00 \\
\hline 41 & $\mathrm{Nb}$ & 92.9060 & 51.906 & 0.75040 & 0.74622 & 1.001 & 0.74868 & $2.72641 \times 10^{15}$ & $5 \times 10^{-4}$ & $2.075 \times 10^{-8}$ & 41.00 \\
\hline 42 & Mo & 95.9420 & 53.940 & 0.71360 & 0.70932 & 1.001 & 0.71181 & $2.72641 \times 10^{15}$ & $5 \times 10^{-4}$ & $2.075 \times 10^{-8}$ & 42.00 \\
\hline 43 & $\mathrm{Tc}$ & 97.9070 & 54.907 & 0.67934 & 0.67509 & 1.003 & 0.67823 & $2.72641 \times 10^{15}$ & $5 \times 10^{-4}$ & $2.075 \times 10^{-8}$ & 43.00 \\
\hline 44 & Ru & 101.070 & 57.070 & 0.64743 & 0.64310 & 1.002 & 0.64591 & $2.72641 \times 10^{15}$ & $5 \times 10^{-4}$ & $2.075 \times 10^{-8}$ & 44.00 \\
\hline 45 & $\mathrm{Rh}$ & 102.905 & 57.905 & 0.61765 & 0.61329 & 1.003 & 0.61639 & $2.72641 \times 10^{15}$ & $5 \times 10^{-4}$ & $2.075 \times 10^{-8}$ & 45.00 \\
\hline 46 & $\mathrm{Pd}$ & 106.421 & 60.420 & 0.58984 & 0.58547 & 1.002 & 0.58810 & $2.72641 \times 10^{15}$ & $5 \times 10^{-4}$ & $2.075 \times 10^{-8}$ & 46.00 \\
\hline 47 & $\mathrm{Ag}$ & 107.868 & 60.868 & 0.56382 & 0.55943 & 1.004 & 0.56276 & $2.72641 \times 10^{15}$ & $5 \times 10^{-4}$ & $2.075 \times 10^{-8}$ & 47.00 \\
\hline 48 & $\mathrm{Cd}$ & 112.411 & 64.411 & 0.53944 & 0.53503 & 1.000 & 0.53723 & $2.72641 \times 10^{15}$ & $5 \times 10^{-4}$ & $2.075 \times 10^{-8}$ & 48.00 \\
\hline 49 & In & 114.818 & 65.818 & 0.51656 & 0.51213 & 1.001 & 0.51460 & $2.72641 \times 10^{15}$ & $5 \times 10^{4}$ & $2.075 \times 10^{-8}$ & 49.00 \\
\hline 50 & $\mathrm{Sn}$ & 118.711 & 68.711 & 0.49062 & 0.49062 & 1.003 & 0.49209 & $2.72641 \times 10^{15}$ & $5 \times 10^{-4}$ & $2.075 \times 10^{-8}$ & 50.00 \\
\hline
\end{tabular}




\begin{tabular}{|c|c|c|c|c|c|c|c|c|c|c|c|}
\hline & & & & & & $\delta$ & & & $k_{1}$ & $k_{2}$ & test \\
\hline 51 & $\overline{\mathrm{Sb}}$ & 1.761 & 70.761 & 337 & 0.47037 & 1.004 & 0.47210 & $2.72641 \times 10^{1}$ & $5 \times 10^{-4} 2$ & s. & 51.0 \\
\hline & $\mathrm{Te}$ & & & & & 1.001 & 70 & & & & 2.0 \\
\hline 3 & I & 126.904 & 7 & 0.43784 & 0.43333 & 0.999 & 13536 & & & & 3.0 \\
\hline$r$ & $\mathrm{Xe}$ & 1.293 & 293 & 41635 & 0.41635 & 1.003 & 41759 & & & & \\
\hline 55 & Cs & 132.905 & .905 & 0180 & 0.40180 & 1.001 & 0.40220 & & & & 5.0 \\
\hline 56 & $\mathrm{Ba}$ & 137.327 & 81.327 & 0.38512 & 0.38512 & 1.005 & 0.38666 & 2.726 & & & 6.0 \\
\hline 57 & $\mathrm{La}$ & 138.905 & 81.905 & 0.37532 & 0.37075 & 0.998 & 0.37266 & & & & 7.0 \\
\hline 58 & $\mathrm{Ce}$ & 140.116 & 8 & 169 & 0.35710 & 1.001 & & & & & 58.0 \\
\hline 59 & Pr & 140 & & & & 1.006 & & & & & 59.0 \\
\hline 60 & $\mathrm{Nd}$ & & & & & & & & & & 0.0 \\
\hline 61 & Pm & 144.910 & 8 & 0.32481 & 0.32481 & 0.998 & 0.32416 & & & & 1.0 \\
\hline 62 & Sm & 150.363 & 3 & 0.31371 & 0.30905 & 1.006 & 232 & & & & 2.0 \\
\hline 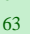 & $\mathrm{Eu}$ & 151.964 & 4 & 313 & 0.30313 & 0.997 & 222 & & & & 3.0 \\
\hline 64 & $\mathrm{Gd}$ & 157.253 & .253 & 0.29305 & 0.28836 & 1.005 & 0.29143 & & & & 4.0 \\
\hline 65 & $\mathrm{~Tb}$ & 158.925 & 25 & 0.2 & 0.27873 & 1.008 & & & & & 5.0 \\
\hline 66 & Dy & 162 & & & & 1.0 & & & & & 5.0 \\
\hline 67 & Ho & 164 & & & & & & & & & .0 \\
\hline 68 & Er & & & & & & & & & & 3.0 \\
\hline 69 & $\mathrm{Tm}$ & 168.934 & 99 & & 0.24435 & 1.005 & & & & & 9.0 \\
\hline 70 & $\mathrm{Yb}$ & 43 & 103.04 & 0 & 0.2 & 1.0 & & & & & 70.0 \\
\hline 1 & $\mathrm{Lu}$ & 57 & 97 & 0 & 0.2 & 0.998 & 62 & & & & 1.0 \\
\hline 72 & $\mathrm{Hf}$ & 17 & & & 0.22223 & 1.007 & & & & & 2.0 \\
\hline 73 & $\mathrm{Ta}$ & 180.948 & 1 & & 0.22030 & 0.999 & & & & & 3.0 \\
\hline 74 & w & & & & & 0.9 & & & & & 1.0 \\
\hline 75 & $\operatorname{Re}$ & 186 & & & 0.2 & 1.000 & & & & & .0 \\
\hline 76 & Os & 19 & & & & & & & & & 5.0 \\
\hline 77 & Ir & & & & & $1 . \mathrm{C}$ & & & & & 7.0 \\
\hline 8 & $\mathrm{Pt}$ & 3 & 08 & 0 & 0 & 18 & & & & & 78.0 \\
\hline 79 & $\mathrm{Au}$ & & & & & 1 & & & & & 9.0 \\
\hline 30 & $\mathrm{Hg}$ & & & & & & & 2.7 & & & \\
\hline 81 & $\mathrm{Tl}$ & 204.383 & 38 & 0.17504 & 0.17504 & 1.003 & & & & & 1.0 \\
\hline 82 & $\mathrm{~Pb}$ & 20 & 20 & & & & & & & & 2.0 \\
\hline 83 & $\mathrm{Bi}$ & 208. & 8 & 0.1 & 0.16 & & & & & & 3.0 \\
\hline 84 & Po & 20 & & & & . & & & & & 4.0 \\
\hline 85 & At & & & & & & & & & & .0 \\
\hline 86 & $\mathrm{Rn}$ & & & & & & & & & & .0 \\
\hline 87 & $\mathrm{Fr}$ & 020 & 02 & & 0.15 & 0.9 & & 2.7 & $5 \times$ & & 87.0 \\
\hline 88 & $\mathrm{Ra}$ & 030 & & 0.14640 & 0.14640 & 0.9 & & 2.72 & & & 8.0 \\
\hline 89 & Ac & 227.030 & 3 & 0.14265 & 0.142 & 1.0 & & & $0^{-4} 2$ & & .0 \\
\hline 90 & Th & 232 & 2.04 & 0.13 & 0.13 & 1.0 & 03 & & & & 0.0 \\
\hline 91 & $\mathrm{~Pa}$ & 23 & & & & & & & & & 1.0 \\
\hline 92 & $\mathrm{U}$ & & & & & & & & & & .0 \\
\hline 93 & $\mathrm{~Np}$ & & & & & & & & & & 8.0 \\
\hline 94 & $\mathrm{Pu}$ & 4.060 & 6 & 0 & 0.1 & 1. & 26 & & ${ }^{4} 2$ & $10^{-8}$ & 4.0 \\
\hline 95 & Am & 3.060 & 05 & 0.12255 & 0.12255 & 1.0 & 365 & & $0^{-4} 2$ & $10^{-8}$ & 95.0 \\
\hline 9 & $\mathrm{Cm}$ & & & & & & & & & & 6.0 \\
\hline 97 & $\mathrm{Bk}$ & & & 0. & 0.1 & 1.0 & 37 & 2.726 & $5 \times 10^{-4} 2$ & $0^{-8}$ & 97.0 \\
\hline 98 & Cf & 251 & 8 & 0 & & 1. & & & & & 8.0 \\
\hline 99 & Es & & 15 & & & 1.020 & & & & & 99.0 \\
\hline \#\# & $\mathrm{Fm}$ & 257.010 & 157.01 & 0.10838 & 0.10838 & 1.021 & 0.11066 & $2.72641 \times 10^{1}$ & $5 \times 10^{-4} 2$ & $2.075 \times 10^{-8}$ & 100.0 \\
\hline
\end{tabular}

\section{Chapter.4. The essence of gravitational mass}

According to above discovery, can get a formula that calculate the atomic mass of element by moving photons, see below formula $m_{a}=\sqrt{\frac{c}{\lambda}+Z Z b} \times\left(1+\left(k_{1} \times \frac{A_{A}}{Z}\right) k_{2}\right.$ Where,

$m_{a}$ is the atomic mass of element, $A_{m}$ is the atomic weight of element, $Z$ is the atomic number or element number, $\lambda_{k 1}$ is the wavelength of X-Ray Emission in lower energy level, $\lambda_{k 2}$ is the wavelength of X-Ray Emission in higher energy level, $\bar{\lambda}$ is the mean wavelength of these two wavelength of X-Ray Emission. $b, k_{1}, k_{2}$ are all constant. For example about $\mathrm{Li}$ :

$m_{a}=\sqrt{\frac{c}{\lambda}+Z b} \times\left(1+\left(k_{1} \times \frac{A_{m}}{Z}\right) k_{2}=\sqrt{\frac{3 \times 10^{8}}{226.456 \times 10^{-10}}+3 \times 2.72641 \times 10^{15}} \times\left(1+1.314 \times \frac{6.941}{3}\right) \times 1.115 \times 10^{-8}=6.5941\right.$

Other atomic mass can also be calculated by this formula. Test about this formula see below Table3, Table $4^{[3],[4],[5],[6]}$.

\begin{tabular}{|c|c|c|c|c|c|c|c|c|c|c|}
\hline & ymb & $\operatorname{vight}\left(A_{n}\right.$ & $A m-Z$ & $\lambda_{\mathrm{s} 1} \times 10^{-10}$ & $\lambda$ & 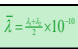 & $b$ & $k_{1}$ & $k_{2}$ & 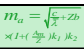 \\
\hline 3 & $\mathrm{Li}$ & 6.941 & 3.941 & & 226.456 & 226.456 & $2.72641 \times 10^{15}$ & 1.314 & $115 \times 1$ & 6.5941 \\
\hline 4 & $\mathrm{Be}$ & 9.012 & 5.012 & & 111.698 & 111.698 & $2.72641 \times 10^{15}$ & 1.314 & $1.115 \times 10^{-8}$ & 8.5812 \\
\hline 5 & B & 10.811 & 5.881 & & 65.9495 & 65.9495 & $2.72641 \times 10^{15}$ & 1.314 & $1.115 \times 10^{-8}$ & 10.413 \\
\hline 6 & C & 12.011 & 6.011 & & 43.6813 & 43.6813 & $2.72641 \times 10^{15}$ & 1.314 & $1.135 \times 10^{-8}$ & 12.01 \\
\hline 1 & $\mathrm{~N}$ & 00 & .000 & & 30.9899 & 30.9899 & $2.72641 \times 10^{15}$ & 1.314 & 1.153 & 14.018 \\
\hline 8 & 0 & .999 & 999 & & 3.3186 & 23.3186 & $2.72641 \times 10^{15}$ & 1.314 & & 15.69 \\
\hline 9 & F & 98 & 998 & & .0894 & 18.0894 & $2.72641 \times 10^{15}$ & 1.314 & $1.115 \times 10^{-8}$ & 18.359 \\
\hline 10 & $\mathrm{Ne}$ & 20.179 & 10.179 & & 1.3023 & 14.3023 & $2.72641 \times 10^{15}$ & 1.314 & $1.135 \times 10^{-8}$ & 20.177 \\
\hline 11 & $\mathrm{Na}$ & 22.989 & 11.989 & & 5693 & 11.5693 & $2.72641 \times 10^{15}$ & 1.314 & $1.135 \times 10^{-8}$ & 911 \\
\hline 12 & $\mathrm{Mg}$ & 305 & 12.305 & 9.51257 & & 9.51257 & $0^{15}$ & 1.314 & & 4.0 \\
\hline 13 & $\mathrm{Al}$ & 6.981 & 3.981 & 7.9484 & & 7.94838 & $2.72641 \times 10^{15}$ & 1.314 & & 26.70 \\
\hline 14 & $\mathrm{Si}$ & 28.085 & 14.085 & & 6.73833 & 6.73833 & $2.72641 \times 10^{15}$ & 1.314 & $1.115 \times 10^{-8}$ & 28.186 \\
\hline 15 & $P$ & 30.973 & 15.973 & 5.78424 & 5.78424 & 5.78424 & $2.72641 \times 10^{15}$ & 1.314 & $1.115 \times 10^{-8}$ & 30.970 \\
\hline 10 & $\mathrm{~S}$ & 32.065 & 16.065 & & 5.03166 & 5.03166 & $41 \times 10^{15}$ & 1.314 & & 32.4 \\
\hline 17 & $\mathrm{Cl}$ & & 18.453 & & & 4.40391 & $2.72641 \times 10^{15}$ & 1.314 & & 35.5 \\
\hline 18 & $\mathrm{Ar}$ & 39.948 & 1.948 & & 3.8861 & 3.8861 & $2.72641 \times 10^{15}$ & 1.314 & $1.115 \times 10^{-8}$ & 39.567 \\
\hline 19 & K & 39.098 & 0.098 & 3.7445 & 3.4365 & 3.5905 & & 1.314 & & 39.078 \\
\hline 20 & $\mathrm{Ca}$ & 40.078 & 0.078 & 3.3617 & 3.0704 & 3.2161 & & 1.314 & $1.115 \times 10^{-8}$ & 40.252 \\
\hline 21 & Sc & 55 & 955 & 3.0343 & 2.7620 & 2.8982 & & 1.314 & & 4.434 \\
\hline 22 & $\mathrm{Ti}$ & & 376 & & 2.4974 & 2.6230 & $2.72641 \times 10^{15}$ & 1.314 & & 47.21 \\
\hline 23 & V & & 941 & 74 & 2.2692 & 2.3883 & $2.72641 \times 10^{15}$ & 1.314 & $10^{-8}$ & 50.069 \\
\hline 24 & $\mathrm{Cr}$ & 51.996 & 27.996 & 2.2897 & 2.0703 & 2.1800 & & 1.314 & & 51.498 \\
\hline 25 & $\mathrm{Mn}$ & 54.938 & 1938 & 2.11 & 1.8965 & 1.99915 & 2.7 & 1.314 & $1.115 \times 10^{-8}$ & 54.291 \\
\hline 26 & $\mathrm{Fe}$ & 55.845 & 45 & 1.5 & 1.7435 & 1.83980 & $1 \times 10^{15}$ & 1.314 & & 5.5 \\
\hline 27 & Co & & & & 1.6082 & 1.69862 & 2.72 & 1.314 & $10^{-8}$ & 58.499 \\
\hline 28 & $\mathrm{Ni}$ & 93 & 30.693 & 18 & 1.4881 & 1.57495 & $0^{15}$ & 1.314 & & 58.921 \\
\hline 29 & $\mathrm{Cu}$ & 63.546 & 34.546 & 1.5444 & 1.3806 & 1.46256 & $2.72641 \times 10^{15}$ & 1.314 & & 63.131 \\
\hline 30 & $\mathrm{Zn}$ & 65.4 & 35.409 & 1.4390 & 1.28339 & 1.36120 & & 1.314 & & 65.152 \\
\hline 31 & Ga & 69.723 & 723 & 1.3401 & 582 & 1.26796 & $41 \times 10^{15}$ & 1.314 & & 99.038 \\
\hline 32 & $\mathrm{Ge}$ & 72.641 & 40 & 41 & 62 & 1.1 & $\times 10^{15}$ & 1.314 & $0^{-8}$ & 2.048 \\
\hline 33 & As & 74.5 & 21 & 1.1799 & 1.04496 & 1.11243 & 2.726 & 1.314 & $1.115 \times 10^{-8}$ & 74.141 \\
\hline 34 & $\mathrm{Se}$ & 78.963 & 4.960 & 1.1048 & 0.97977 & 1.04229 & & 1.314 & & 78.078 \\
\hline 35 & $\mathrm{Br}$ & .904 & 44.904 & 1.0438 & 0.92045 & 0.98213 & & 1.314 & & 79.366 \\
\hline 36 & $\mathrm{Kr}$ & & 798 & 801 & 6555 & 0.92283 & $0^{15}$ & 1.314 & & 83.038 \\
\hline 37 & $\mathrm{Rb}$ & 85.467 & .467 & 2558 & 81556 & 0.87057 & 2.72 & 1.314 & $1.120 \times 10^{-8}$ & 85.115 \\
\hline 38 & $\mathrm{Sr}$ & 87.621 & 49.620 & 0.8795 & 0.76976 & 0.82463 & 2.72 & 1.314 & $1.118 \times$ & 87.148 \\
\hline 39 & Y & 05 & 49.905 & 8330 & 0.72769 & 0.78035 & & 1.314 & 1.11 & 88.786 \\
\hline 40 & $\mathrm{Zr}$ & .224 & 51.224 & 7901 & 0.68885 & 0.73948 & & 1.314 & & 91.201 \\
\hline 41 & $\mathrm{Nb}$ & & & & & 170 & 2.72 & 1.314 & $0^{-8}$ & 391 \\
\hline 42 & Mo & & 40 & & 0.6 & 0.66677 & 2.7 & 1.314 & $0^{-8}$ & 95.838 \\
\hline 43 & $\mathrm{Tc}$ & 97.907 & 1907 & 793 & 0.58908 & 0.63419 & & 1.314 & & 97.997 \\
\hline 44 & $\mathrm{Ru}$ & 101.07 & . 070 & 474 & 0.56053 & 0.60397 & & 1.314 & & 101.027 \\
\hline 45 & $\mathrm{Rh}$ & 2.905 & 57.905 & 176 & 0.54121 & 0.57941 & 2.7 & 1.314 & $10^{-8}$ & 22.804 \\
\hline 46 & $\mathrm{Pd}$ & & & & & & 2.72 & 1.314 & 1.11 & \\
\hline 47 & $\mathrm{Ag}$ & 107.868 & 60.868 & 38 & 0.49771 & 0.53076 & 2.7 & 1.314 & $0^{-8}$ & 107.650 \\
\hline 48 & $\mathrm{Cd}$ & 41 & 4.411 & 5394 & 0.46409 & 0.50175 & 2.7 & 1.314 & & 112.372 \\
\hline 49 & In & 4.818 & 8.818 & 5165 & 44372 & 0.48011 & & 1.314 & & 14.897 \\
\hline 50 & Sn & & 68.711 & & & & 2.7 & 1.314 & $1.115 \times 10^{-8}$ & 47 \\
\hline 51 & $\mathrm{Sb}$ & & 61 & 48 & 0.40669 & & 2.7 & 1.314 & & 121.572 \\
\hline 52 & $\mathrm{Te}$ & 7.603 & 5.603 & 0.4513 & 0.38975 & 0.42053 & 2.7 & 1.314 & & 127.051 \\
\hline 53 & I & 126.904 & 3.904 & .78 & 0.37383 & 0.40582 & & 1.314 & & 126.9 \\
\hline 54 & $\mathrm{Xe}$ & 1.293 & 293 & 208 & 0.35844 & 62 & & 1.314 & & 131.020 \\
\hline 55 & Cs & 2.9003 & 77.905 & 4048 & 0.34453 & 0.37467 & & 1.314 & $1.115 \times 10^{-8}$ & 32.959 \\
\hline 56 & $\mathrm{Ba}$ & 7.327 & 81.327 & 3896 & 0.33105 & 0.36033 & 2.72 & 1.314 & & 7.082 \\
\hline 57 & $\mathrm{La}$ & & 05 & & & & 2.72 & 1.314 & & 8.931 \\
\hline 58 & $\mathrm{Ce}$ & & 16 & & 49 & & $0^{15}$ & 1.314 & $10^{-8}$ & 0.695 \\
\hline 59 & Pr & & 07 & & 498 & 684 & $0^{15}$ & 1.314 & $10^{-8}$ & 140.890 \\
\hline 60 & $\mathrm{Nd}$ & 4.243 & 240 & 3365 & 29404 & 527 & 2.7 & 1.314 & $10^{-8}$ & 144.270 \\
\hline 61 & $\mathrm{Pm}$ & & 910 & 0.3248 & 61 & 21 & 2.7 & 1.314 & $10^{-8}$ & 45.524 \\
\hline 62 & Sm & 150.363 & 363 & 0.3137 & 0.27376 & 0.29373 & & 1.314 & $10^{-8}$ & 150.418 \\
\hline 63 & Eu & 151.964 & .964 & 0.3031 & 26434 & 0.28372 & & 1.314 & $<10^{-8}$ & 151.987 \\
\hline 64 & $\mathrm{Gd}$ & 157.253 & 53 & 0.2930 & 535 & 18 & & 1.314 & & 57.200 \\
\hline 65 & $\mathrm{~Tb}$ & 5 & 93.925 & & 684 & 12 & & 1.314 & $1.113 \times 10^{-8}$ & 158.954 \\
\hline 66 & Dy & 62.500 & 500 & 2743 & 0.23863 & 0.25647 & $10^{15}$ & 1.314 & $1.115 \times 10^{-8}$ & 162.745 \\
\hline 67 & Но & 164. & 7.930 & 655 & 0.23084 & 0.2 & $\times 10^{15}$ & 1.314 & $1.112 \times 10^{-8}$ & 54.952 \\
\hline 68 & Er & 167 & 9.259 & 72 & 42 & & $1 \times 10^{15}$ & 1.314 & $.115 \times 10^{-8}$ & 7.957 \\
\hline 69 & $\mathrm{Tm}$ & 168. & 34 & 2491 & 0.2 & 73 & $2.72641 \times 10^{15}$ & 1.314 & $1.112 \times 10^{-8}$ & 9.589 \\
\hline 70 & $\mathrm{Yb}$ & & 04 & & 0. & & $\times 10^{15}$ & 1.314 & $10^{-8}$ & 73.409 \\
\hline 71 & Lu & & & & & & $10^{15}$ & 1.314 & & 75.689 \\
\hline 7 & $\mathrm{Hf}$ & & & & & & & 1.314 & & 19.20 \\
\hline 73 & Ta & & 95 & & & & & 1.314 & $10^{-8}$ & 31.916 \\
\hline 74 & W & & & & & & & 1.314 & $\times 10^{-8}$ & 34.993 \\
\hline 75 & $\operatorname{Re}$ & & & & 35 & & & 1.314 & $1.115 \times 10^{-8}$ & 37.653 \\
\hline 76 & Os & & 4.20 & & 6 & & 2.72 & 1.314 & $1.115 \times 10^{-8}$ & 1.579 \\
\hline 77 & Ir & 0 & .22 & & 0.1 & 0.18388 & $2.72641 \times 10^{15}$ & 1.314 & $1.112 \times 10^{-8}$ & 193.482 \\
\hline 78 & $\mathrm{Pt}$ & 195.080 & 117.08 & 1921 & 0.16509 & 0.17865 & $2.72641 \times 10^{15}$ & 1.314 & $1.112 \times 10^{-8}$ & 196.555 \\
\hline 79 & $\mathrm{Au}$ & 77 & 17.97 & & 0.18020 & 20 & $2.72641 \times 10^{15}$ & 1.314 & $1.115 \times 10^{-8}$ & 5.795 \\
\hline 00 & $\mathrm{Hg}$ & & 0.59 & & 507 & 0.17507 & $2.72641 \times 10^{15}$ & 1.314 & $1.118 \times 10^{-8}$ & 0.020 \\
\hline 81 & $\mathrm{Tl}$ & & 1.38 & & 0.17013 & 0.17013 & $2.72641 \times 10^{15}$ & 1.314 & $1.115 \times 10^{-8}$ & 03.322 \\
\hline 82 & $\mathrm{~Pb}$ & & 125.20 & & 38 & 0.16538 & & 1.314 & $1.115 \times 10^{-8}$ & 206.425 \\
\hline 83 & $\mathrm{Bi}$ & & 125.98 & & 79 & . 16079 & & 1.314 & & 208.757 \\
\hline 84 & Po & & 4.98 & & 6633 & 33 & & 1.314 & $1.115 \times 10^{-8}$ & 209.76 \\
\hline 85 & At & & & & & & $\times 10^{15}$ & 1.314 & $1.115 \times 10^{-8}$ & 211.56 \\
\hline
\end{tabular}




\begin{tabular}{|c|c|c|c|c|c|c|c|c|c|c|}
\hline \multicolumn{3}{|c|}{ Z symbolatomic weight $\left(A_{m}\right)$} & \multirow{2}{*}{$\frac{{ }^{A m-Z}}{136.02}$} & $\lambda_{k 1} \times 10^{-10}$ & \multirow{2}{*}{$\begin{array}{l}\lambda_{12} \times 10^{-10} \\
0.14797\end{array}$} & \multirow{2}{*}{$\frac{\bar{\lambda}=\frac{1+2}{2} \times 10^{-11}}{0.14797}$} & \multirow{2}{*}{$\begin{array}{c}b \\
2.72641 \times 10^{15}\end{array}$} & \multirow{2}{*}{$\begin{array}{l}k_{l} \\
1.314\end{array}$} & \multirow{2}{*}{$\frac{k_{2}}{1.115 \times 10^{-8}}$} & \multirow{2}{*}{ 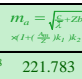 } \\
\hline 86 & $\mathrm{Rn}$ & 222.017 & & & & & & & & \\
\hline 87 & $\mathrm{Fr}$ & 223.019 & 136.02 & & 0.14399 & 0.14399 & $2.72641 \times 10^{15}$ & 1.314 & $1.115 \times 10^{-8}$ & 223.586 \\
\hline 88 & $\mathrm{Ra}$ & 226.025 & 138.03 & & 0.14014 & 0.14014 & $2.72641 \times 10^{15}$ & 1.314 & $1.115 \times 10^{-8}$ & 226.959 \\
\hline 89 & $\mathrm{Ac}$ & 7.028 & 138.03 & 0.1414 & 0.13640 & 0.13890 & $2.72641 \times 10^{15}$ & 1.314 & $1.118 \times 10^{-8}$ & 227.379 \\
\hline 90 & Th & 232.038 & 142.04 & 0.1378 & 0.13282 & 32 & 2.7264 & 1.314 & $10^{-8}$ & 1.625 \\
\hline 91 & $\mathrm{~Pa}$ & 232.0 & 141.04 & 0.1344 & 0.12933 & 84 & 2.72 & 1.314 & & 652 \\
\hline 92 & U & 238.028 & 146.03 & 0.1310 & 0.12595 & 0.12848 & 2.726 & 1.314 & & (2 \\
\hline 93 & $\mathrm{~Np}$ & 237.048 & 144.05 & 0.1288 & 0.12268 & 0.12575 & $2.72641 \times 10^{15}$ & 1.314 & $1.112 \times 10^{-8}$ & 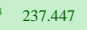 \\
\hline 94 & $\mathrm{Pu}$ & 244.06 & 150.06 & 0.1246 & 0.11951 & 0.12204 & $2.72641 \times 10^{15}$ & 1.314 & $1.115 \times 10^{-8}$ & 156 \\
\hline 95 & $\mathrm{Am}$ & 243. & 149.06 & 0.12 & & 0.12150 & $2.72641 \times$ & 1.314 & $1.115 \times 10^{-8}$ & 935 \\
\hline 96 & $\mathrm{Cm}$ & 247. & 151.07 & 0.1185 & & 54 & $0^{15}$ & 1.314 & $10^{-8}$ & 7.051 \\
\hline 91 & $\mathrm{Bk}$ & 247 & 150.07 & & & 0.11566 & 2.726 & 1.314 & $\times 10^{-8}$ & 248.101 \\
\hline 98 & $\mathrm{Cf}$ & 251. & 153.08 & & 0.11288 & 0.11288 & $2.72641 \times 10^{15}$ & 1.314 & $1.112 \times 10^{-8}$ & 251.572 \\
\hline 99 & Es & 252.083 & 153.08 & & & 0.11107 & $2.72641 \times 10^{15}$ & 1.314 & $1.112 \times 10^{-8}$ & 252.387 \\
\hline 100 & $\mathrm{Fm}$ & 257.095 & 157.09 & 0.1084 & & 0.10838 & $2.72641 \times 10^{15}$ & 1.314 & $1.112 \times 10^{-8}$ & 257.33 \\
\hline
\end{tabular}

In these tables: Some data got by formula: $=\frac{12398.418}{E[e \mathrm{~V}]}$. This calculated show that the essence of gravitational mass is due to the photons moving. Because the atomic mass in the periodic table of elements is the weighted aggregates, for accurately test the meaning of $M_{a}=\sqrt{\frac{C}{\bar{\lambda}}+n b}\left(1+1.314 \times \frac{A_{m}}{z}\right) k$, see below test: Table $5^{[3],[4],[5],[6]}$

\begin{tabular}{|c|c|c|c|c|c|c|c|c|c|}
\hline Z & symbo & ic weight & ural abundance & $\lambda_{k 1} \times 10^{-10}$ & $\lambda_{k 2} \times 10^{-10}$ & $\bar{\lambda}=\frac{4+2 \times}{2} \times 10^{-11}$ & $b$ & $k_{l}$ & 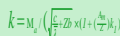 \\
\hline 3 & $\mathrm{Li}$ & 7.012 & 92.414 & & 226.456 & 226.456 & $2.72641 \times 10^{15}$ & 1.314 & $1.17661 \times 10^{-8}$ \\
\hline 4 & $\mathrm{Be}$ & 9.012 & 100 & & 111.698 & 111.698 & $2.72641 \times 10^{15}$ & 1.314 & $1.17095 \times 10^{-8}$ \\
\hline 5 & B & 11.01 & 80.17 & 65.9496 & & 65.9496 & $2.72641 \times 10^{15}$ & 1.314 & $1.16525 \times 10^{-8}$ \\
\hline 6 & $\mathrm{C}$ & 12.00 & 98.93 & & 43.6813 & 43.6813 & $2.72641 \times 10^{15}$ & 1.314 & $1.13425 \times 10^{-8}$ \\
\hline 7 & $\mathrm{~N}$ & 14.00 & 99.636 & & 30.9899 & 30.9899 & $2.72641 \times 10^{15}$ & 1.314 & $1.13353 \times 10^{-8}$ \\
\hline 8 & 0 & 16.00 & 99.757 & & 23.3186 & 23.3186 & $2.72641 \times 10^{15}$ & 1.314 & $1.13686 \times 10^{-8}$ \\
\hline 9 & $\mathrm{~F}$ & 19.00 & 100 & & 18.0894 & 18.0894 & $2.72641 \times 10^{15}$ & 1.314 & $1.15379 \times 10^{-8}$ \\
\hline 10 & $\mathrm{Ne}$ & 19.99 & 90.48 & & 14.3023 & 14.3023 & $2.72641 \times 10^{15}$ & 1.314 & $1.13220 \times 10^{-8}$ \\
\hline 11 & $\mathrm{Na}$ & 22.99 & 100 & & 11.5755 & 11.5755 & $2.72641 \times 10^{15}$ & 1.314 & $1.14121 \times 10^{-8}$ \\
\hline 12 & $\mathrm{Mg}$ & 25.99 & 10.029 & 9.8903 & & 9.89030 & $2.72641 \times 10^{15}$ & 1.314 & $1.16570 \times 10^{-8}$ \\
\hline 13 & $\mathrm{Al}$ & 26.98 & 100 & 8.3420 & & 8.34200 & $2.72641 \times 10^{15}$ & 1.314 & $1.15171 \times 10^{-8}$ \\
\hline 14 & $\mathrm{Si}$ & 27.98 & 92.223 & & 7.0665 & 7.06650 & $2.72641 \times 10^{15}$ & 1.314 & $1.13433 \times 10^{-8}$ \\
\hline 15 & P & 30.97 & 100 & 6.1661 & 6.1088 & 6.13345 & $2.72641 \times 10^{15}$ & 1.314 & $1.14575 \times 10^{-8}$ \\
\hline 16 & S & 31.97 & 94.99 & 5.3719 & 5.0187 & 5.19530 & $2.72641 \times 10^{15}$ & 1.314 & $1.11894 \times 10^{-8}$ \\
\hline 17 & $\mathrm{Cl}$ & 34.97 & 75.76 & 4.8889 & 4.3982 & 4.64350 & $2.72641 \times 10^{15}$ & 1.314 & $1.13490 \times 10^{-8}$ \\
\hline 18 & Ar & 39.96 & 99.603 & 4.1948 & 3.8710 & 4.03290 & $2.72641 \times 10^{15}$ & 1.314 & $1.14563 \times 10^{-8}$ \\
\hline 19 & K & 38.96 & 93.258 & 3.7445 & 3.4365 & 3.59050 & $2.72641 \times 10^{15}$ & 1.314 & $1.11955 \times 10^{-8}$ \\
\hline 20 & $\mathrm{Ca}$ & 39.96 & 96.941 & 3.3617 & & 3.36170 & $2.72641 \times 10^{15}$ & 1.314 & $1.13272 \times 10^{-8}$ \\
\hline 21 & Sc & 44.96 & 100 & 3.0343 & 2.7620 & 2.8982 & $2.72641 \times 10^{15}$ & 1.314 & $1.12808 \times 10^{-8}$ \\
\hline 22 & $\mathrm{Ti}$ & 47.95 & 73.72 & 2.7523 & 2.4974 & 2.6249 & $2.72641 \times 10^{15}$ & 1.314 & $1.13147 \times 10^{-8}$ \\
\hline 23 & V & 50.94 & 99.75 & 2.5074 & 2.2692 & 2.3883 & $2.72641 \times 10^{15}$ & 1.314 & $1.13441 \times 10^{-8}$ \\
\hline 24 & $\mathrm{Cr}$ & 51.94 & 83.789 & 2.2936 & 2.0703 & 2.1820 & $2.72641 \times 10^{15}$ & 1.314 & $1.12596 \times 10^{-8}$ \\
\hline 25 & Mn & 54.94 & 100 & 2.1058 & 1.8965 & 2.0012 & $2.72641 \times 10^{15}$ & 1.314 & $1.12883 \times 10^{-8}$ \\
\hline 26 & $\mathrm{Fe}$ & 55.93 & 91.754 & 1.9401 & 1.7435 & 1.8418 & $2.72641 \times 10^{15}$ & 1.314 & $1.12098 \times 10^{-8}$ \\
\hline 27 & Сo & 58.93 & 100 & 1.7929 & 1.6082 & 1.70055 & $2.72641 \times 10^{15}$ & 1.314 & $1.12388 \times 10^{-8}$ \\
\hline 28 & $\mathrm{Ni}$ & 57.94 & 68.077 & 1.6618 & & 1.66180 & $2.72641 \times 10^{15}$ & 1.314 & $1.13572 \times 10^{-8}$ \\
\hline 29 & $\mathrm{Cu}$ & 62.93 & 69.15 & 1.5444 & 1.3806 & 1.46256 & $2.72641 \times 10^{15}$ & 1.314 & $1.11949 \times 10^{-8}$ \\
\hline 30 & $\mathrm{Zn}$ & 65.93 & 27.73 & 1.4390 & 1.28339 & 1.36120 & $2.72641 \times 10^{15}$ & 1.314 & $1.12167 \times 10^{-8}$ \\
\hline 31 & Ga & 68.93 & 60.108 & 1.3440 & 1.19582 & 1.26991 & $2.72641 \times 10^{15}$ & 1.314 & $1.12360 \times 10^{-8}$ \\
\hline 32 & Ge & 73.92 & 36.521 & 1.2580 & 1.11662 & 1.18731 & $2.72641 \times 10^{15}$ & 1.314 & $1.13301 \times 10^{-8}$ \\
\hline 33 & As & 74.92 & 100 & 1.1799 & 1.04496 & 1.11243 & $2.72641 \times 10^{15}$ & 1.314 & $1.12673 \times 10^{-8}$ \\
\hline 34 & $\mathrm{Se}$ & 79.92 & 49.803 & 1.1088 & 0.97977 & 1.04430 & $2.72641 \times 10^{15}$ & 1.314 & $1.13722 \times 10^{-8}$ \\
\hline 35 & $\mathrm{Br}$ & 80.92 & 49.31 & 1.0438 & 0.92045 & 0.98213 & $2.72641 \times 10^{15}$ & 1.314 & $1.12909 \times 10^{-8}$ \\
\hline 36 & Kr & 83.91 & 56.987 & 0.9841 & 0.86555 & 0.92483 & $2.72641 \times 10^{15}$ & 1.314 & $1.12978 \times 10^{-8}$ \\
\hline 37 & $\mathrm{Rb}$ & 84.91 & 72.172 & 0.9297 & 0.81556 & 0.87263 & $2.72641 \times 10^{15}$ & 1.314 & $1.12408 \times 10^{-8}$ \\
\hline 38 & $\mathrm{Sr}$ & 87.91 & 82.581 & 0.8795 & 0.76976 & 0.82463 & $2.72641 \times 10^{15}$ & 1.314 & $1.12497 \times 10^{-8}$ \\
\hline 39 & Y & 88.91 & 100 & 0.8330 & 0.72769 & 0.78035 & $2.72641 \times 10^{15}$ & 1.314 & $1.11950 \times 10^{-8}$ \\
\hline 40 & $\mathrm{Zr}$ & 89.90 & 51.45 & 0.7901 & 0.68885 & 0.73948 & $2.72641 \times 10^{15}$ & 1.314 & $1.11418 \times 10^{-8}$ \\
\hline 41 & $\mathrm{Nb}$ & 92.91 & 100 & 0.7504 & 0.65300 & 0.70170 & $2.72641 \times 10^{15}$ & 1.314 & $1.11517 \times 10^{-8}$ \\
\hline 42 & Mo & 97.91 & 24.292 & 0.7136 & 0.61994 & 0.66677 & $2.72641 \times 10^{15}$ & 1.314 & $1.12182 \times 10^{-8}$ \\
\hline 43 & Tc & 99.91 & $15.8 \mathrm{~s}$ & 0.6793 & 0.58908 & 0.63419 & $2.72641 \times 10^{15}$ & 1.314 & $1.11958 \times 10^{-8}$ \\
\hline 44 & $\mathrm{Ru}$ & 101.90 & 31.550 & 0.6474 & 0.56053 & 0.60397 & $2.72641 \times 10^{15}$ & 1.314 & $1.11745 \times 10^{-8}$ \\
\hline 45 & $\mathrm{Rh}$ & 102.91 & 100 & 0.6176 & 0.53396 & 0.57578 & $2.72641 \times 10^{15}$ & 1.314 & $1.11267 \times 10^{-8}$ \\
\hline 46 & Pd & 107.90 & 26.46 & 0.5898 & 0.50922 & 0.54951 & $2.72641 \times 10^{15}$ & 1.314 & $1.11848 \times 10^{-8}$ \\
\hline 47 & $\mathrm{Ag}$ & 108.91 & 48.162 & 0.5638 & 0.48590 & 0.52485 & $2.72641 \times 10^{15}$ & 1.314 & $1.11405 \times 10^{-8}$ \\
\hline 48 & $\mathrm{Cd}$ & 113.90 & 28.754 & 0.5394 & 0.46409 & 0.50175 & $2.72641 \times 10^{15}$ & 1.314 & $1.11897 \times 10^{-8}$ \\
\hline 49 & In & 114.90 & 95.715 & 0.5165 & 0.44372 & 0.48011 & $2.72641 \times 10^{15}$ & 1.314 & $1.11443 \times 10^{-8}$ \\
\hline 50 & $\mathrm{Sn}$ & 119.90 & 32.589 & 0.4950 & 0.42468 & 0.45984 & $2.72641 \times 10^{15}$ & 1.314 & $1.11924 \times 10^{-8}$ \\
\hline
\end{tabular}

Table $6^{[3],[4],[5],[6]}$ :

\begin{tabular}{|c|c|c|c|c|c|c|c|c|c|}
\hline$Z$ & symt & & & $10^{-10}$ & $\lambda_{k 2} \times 10^{-10}$ & $\bar{\lambda}=\frac{1-4}{2} \times 10^{-11}$ & $b$ & $k_{l}$ & 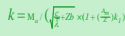 \\
\hline 51 & $\mathrm{Sb}$ & 122.904 & 42.79 & 0.4748 & 0.40669 & 0.44075 & $2.72641 \times 10^{15}$ & 1.31 & $1.11925 \times 10^{-8}$ \\
\hline 52 & $\mathrm{Te}$ & 125.903 & 18.84 & 0.4558 & 0.38975 & 0.42278 & $2.72641 \times 10^{15}$ & 1.31 & $1.11920 \times 10^{-8}$ \\
\hline 33 & I & 126.904 & 100 & 0.4378 & 0.37383 & 0.40582 & $2.72641 \times 10^{15}$ & 1.31 & $1.11486 \times 10^{-8}$ \\
\hline 54 & $\mathrm{Xe}$ & 128.904 & 26.908 & 0.4208 & 0.35844 & 0.38962 & $2.72641 \times 10^{15}$ & 1.31 & $.11239 \times 10^{-8}$ \\
\hline 55 & $\mathrm{Cs}$ & 132.905 & 100 & 0.4048 & 0.34453 & 0.37467 & $2.72641 \times 10^{15}$ & 1.31 & $1.11454 \times 10^{-8}$ \\
\hline 56 & $\mathrm{Ba}$ & 137.905 & 71.698 & 0.3896 & 0.33105 & 0.36033 & $2.72641 \times 10^{15}$ & 1.31 & \\
\hline 57 & $\mathrm{La}$ & 138.905 & 99.911 & 0.3753 & 0.31845 & 0.34688 & $2.72641 \times 10^{15}$ & 1.31 & $.11406 \times 10^{-8}$ \\
\hline 58 & $\mathrm{Ce}$ & 141.909 & 11.114 & 0.3617 & 0.30649 & 0.33410 & $2.72641 \times 10^{15}$ & 1.31 & $1.11378 \times 10^{-8}$ \\
\hline 59 & Pr & 140.907 & 100 & 0.3487 & 0.30498 & 0.32684 & $2.72641 \times 10^{15}$ & 1.31 & $19 \times 10^{-8}$ \\
\hline 60 & $\mathrm{Nd}$ & 145.913 & 17.189 & 0.3365 & 0.29404 & 0.31527 & $2.72641 \times 10^{15}$ & 1.31 & $86 \times 10^{-8}$ \\
\hline 61 & $\mathrm{Pm}$ & 147.917 & $37 \mathrm{~d}$ & 0.3248 & 0.28361 & 0.30421 & $2.72641 \times 10^{15}$ & 1.31 & $79 \times 10^{-8}$ \\
\hline 62 & $\mathrm{Sm}$ & 151.919 & .75 & 0.3137 & 0.27376 & 0.29373 & $2.72641 \times 10^{15}$ & 1.31 & $32 \times 10^{-8}$ \\
\hline 63 & Eu & 152.921 & 55.196 & 0.3031 & 0.26434 & 0.28372 & $2.72641 \times 10^{15}$ & 1.31 & $50 \times 10^{-8}$ \\
\hline 64 & $\mathrm{Gd}$ & 157.924 & 24.84 & 0.2930 & 0.25535 & 0.27418 & $2.72641 \times 10^{15}$ & 1.31 & $\times 10^{-8}$ \\
\hline 65 & $\mathrm{~Tb}$ & 158.925 & 100 & 0.2834 & 0.24684 & 0.26512 & $2.72641 \times 10^{15}$ & 1.31 & $9 \times 10^{-8}$ \\
\hline 66 & Dy & 163.929 & 28.260 & 0.2743 & .23863 & 0.25647 & $2.72641 \times 10^{15}$ & 1.31 & $1 \times 10^{-8}$ \\
\hline 67 & Но & 164.930 & 100.000 & 0.2655 & 0.23084 & 0.24817 & $2.72641 \times 10^{15}$ & 1.31 & \\
\hline 68 & Er & 167.932 & 978 & 0.2572 & 0.22342 & 0.24031 & $2.72641 \times 10^{15}$ & 1.31 & $1 \times 10^{-8}$ \\
\hline 69 & $\mathrm{Tm}$ & 168.934 & 100 & 0.2491 & & 0.24910 & $2.72641 \times 10^{15}$ & 1.31 & $2 \times 10^{-8}$ \\
\hline 70 & $\mathrm{Yb}$ & 173.938 & 32.026 & 0.2438 & 0.21037 & 0.22708 & $2.72641 \times 10^{15}$ & 1.31 & $99 \times 10^{-8}$ \\
\hline 71 & Lu & 174.967 & 97.40 & 0.2364 & 0.20384 & 0.22012 & $2.72641 \times 10^{15}$ & 1.31 & $38 \times 10^{-8}$ \\
\hline 72 & $\mathrm{Hf}$ & 1799.946 & 35.08 & 0.2293 & 0.19759 & 0.21345 & $2.72641 \times 10^{15}$ & 1.31 & $67 \times 10^{-8}$ \\
\hline 73 & Та & 180.948 & 99.99 & 0.2225 & & 0.22245 & $2.72641 \times 10^{15}$ & 1.31 & $9 \times 10^{-8}$ \\
\hline 74 & w & 183.950 & 30.64 & 0.2159 & & 0.21592 & $2.72641 \times 10^{15}$ & 1.31 & $6 \times 10^{-8}$ \\
\hline 75 & $\operatorname{Re}$ & 186.955 & 62 & 0.209 & & 960 & $2.72641 \times 10^{15}$ & 1.3. & $5 \times 10^{-8}$ \\
\hline 76 & Os & & & 0. & & & $41 \times 10^{15}$ & 1. & $10^{-8}$ \\
\hline 77 & Ir & 19 & ( & 0.197 & & 19780 & $641 \times 10^{15}$ & 1.31 & $8 \times 10^{-8}$ \\
\hline 78 & $\mathrm{Pt}$ & 195.96 & 25.21 & 0.1921 & & 19214 & $2.72641 \times 10^{15}$ & 1.31 & $\times 10^{-8}$ \\
\hline 79 & $\mathrm{Au}$ & 196. & 100.00 & & 0. & 20 & & 1.3. & $\times 10^{8}$ \\
\hline 80 & $\mathrm{Hg}$ & & & & & & & 1. & $3 \times 10^{-8}$ \\
\hline 81 & $\mathrm{Tl}$ & 204.974 & 70.48 & & 13 & 17013 & $2.72641 \times 10^{15}$ & 1. & $7 \times 10^{-8}$ \\
\hline 82 & $\mathrm{~Pb}$ & 76 & 52 & & 6538 & 16538 & $2.72641 \times 10^{15}$ & 1.3 & $5 \times 10^{-8}$ \\
\hline 83 & $\mathrm{Bi}$ & 26 & 100.00 & & 79 & 0.11 & $2.72641 \times 10^{15}$ & 1. & $1.11412 \times 10^{-8}$ \\
\hline 84 & Po & & & & & & $0^{15}$ & 1.3 & $0 \times 10^{-8}$ \\
\hline 85 & At & 21 & & & & 09 & & 1.31 & \\
\hline 86 & $\mathrm{Rn}$ & & 3.8 & & & & & 1.31 & $\times 10^{-8}$ \\
\hline 87 & Fr & & & & & & & 1.31 & $2 \times 10^{-8}$ \\
\hline 88 & $\mathrm{Ra}$ & & & & & & $10^{15}$ & 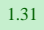 & $4 \times 10^{-8}$ \\
\hline 89 & $\mathrm{Ac}$ & & & 0.1414 & 40 & 90 & & 1.31 & $2 \times 10^{-8}$ \\
\hline 30 & Th & 232. & 99.98 & 0.1378 & 0.13282 & 0.13532 & & 1.31 & $8 \times 10^{-8}$ \\
\hline 91 & $\mathrm{~Pa}$ & & & 0.13 & & & & 1.31 & \\
\hline 92 & $\mathrm{U}$ & & & & & & & & \\
\hline 93 & $\mathrm{~Np}$ & & & 0.12 & & & $641 \times 10^{15}$ & 1.31 & $39 \times 10^{-8}$ \\
\hline 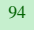 & $\mathrm{Pu}$ & 246. & $10.85 \mathrm{~d}$ & 0.1246 & & 457 & 2.72 & 1.31 & $12343 \times 10^{-8}$ \\
\hline 05 & $\mathrm{Am}$ & 247. & & 0.1215 & & 150 & & 1.31 & \\
\hline 96 & $\mathrm{Cm}$ & & & & & & & 131 & $.11508 \times 10^{-8}$ \\
\hline 97 & $\mathrm{Bk}$ & & $320 \mathrm{~d}$ & & & & $2.72641 \times 10^{15}$ & 1.31 & $1.11243 \times 10^{-8}$ \\
\hline 98 & Cf & 254. & $60.5 \mathrm{~d}$ & & 0.11288 & 0.11288 & & 1.31 & $1.11283 \times 10^{-8}$ \\
\hline 99 & Es & 255 & 40 & 0.11 & & 0.1 & & 1.3 & $1.11359 \times 10^{-8}$ \\
\hline 00 & $\mathrm{Fm}$ & 259.101 & 1. & 0.1084 & & 0.10838 & $10^{15}$ & 1.31 & \\
\hline
\end{tabular}

In this table: the calculated wavelength is considered by emit from the atom of the most abundance in nature. The wavelength of X-ray corresponds to one atomic mass for accurate result, but not its average atomic mass which used in table 5 and table 6 .

\section{Chapter.5. Unification of gravity and electromagnetic force}

From below formulas:

$Q_{Z}=\sqrt{\frac{c}{\bar{\lambda}}+Z b} \times\left(1-\left(A_{m}-Z\right) k_{1}\right) \times k_{2}$

$m_{a}=\sqrt{\frac{c}{\lambda}+Z b} \times\left(1+k_{1} \times \frac{A_{m}}{Z}\right) \times k_{2}{ }^{[2]}$ we can see

below interaction between two unclears of lithium, between them, the size of their Coulomb force is:

$F_{c}=\frac{Q_{1} \times Q_{2}}{r^{2}} k=\frac{\left[\sqrt{\frac{c}{x}+Z b} \times\left(1-\left(A_{m}-Z\right) k_{l}\right) \times k_{2}\right]^{2}}{r^{2}} k=\frac{\left(\frac{c}{\lambda}+Z b\right)}{r^{2}} k \times\left[\left(1-\left(A_{m}-Z\right) k_{1}\right) \times k_{2}\right]^{2}$

Between them the size of their gravitational force

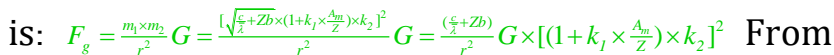
above, we can know the electromagnetic force and 
gravitational force in atom are all produced by the moving photons in atom, so their origins are same: $\frac{\left(\frac{c}{i}+Z b\right)}{r^{2}}$.the difference size of value between them is only due to the Coulomb force is the first interactional force by this produced force, it do not include gravitational force, the gravitational force include the effect of Coulomb force interacted: $\left(1-\left(\mathrm{A}_{m}-Z\right) k_{1}\right) \times k_{2},\left(1+k_{1} \times \frac{A_{m}}{Z}\right) \times k_{2}$.

Now we have preliminary known the unification of the electromagnetic force and gravitational force ${ }^{[5-6]}$ first time .

\section{Chapter.6. Conclusion}

We have known the essence of electric charge and the essence of gravitational mass in atom; meanwhile we have also known the unification of gravitation and electromagnetic force by the way of applying new discoveries, about the more contents and the more evidences of unification of the electromagnetic force and gravitational force, please waiting to see will be coming next article.

\section{Acknowledgment}

I express my appreciation sincerely to Yang Zhenfeng who is in the institute of Mathematic, and my friend Shao shengtang, Jiu guoqiang, Wang xuezheng, Xi xinghua, and Dong qianlin, my middle school teacher Zhang linxiang. My young uncle sister Gou susheng. My young brother Zheng guoman, My young sister Zhnegjunfang.They all gave me a lot of spiritual and financial support when I did the research about this paper. I also express my gratitude for my father Zheng heizi, who gave me great love, and that is the driving force of my moving forward.

\section{References}

[1] Isaac Newton philosophiae naturalis principia mathematuca praeses julii ,5,1687 londini

[2] Zheng shengming, Physicist (2021): Grativation origin. TechRxiv. Preprint. https://doi.org/10.36227/techrxiv.16677298.v1

[3] Günter Zschomarch Handbook of X-Ray Data, springer 2007

[4] E.W.White.G.G.Johnson, Jr X-Ray emission and absorption edge wavelengths. The Pennsylvania state university.
[5] Zheng sheng ming. Mechanism of interaction in moving matter-the origin of gravitation. ISBN 9783847326588.

[6] Zheng sheng ming. Nature mechanism-force origin. ISBN 97898819868632012. 